\title{
Punção folicular guiada por ultra-sonografia em éguas tratadas com FSH no estroma ovariano: avaliação da taxa de recuperação dos ovócitos
}

\section{Follicular puncture guided by ultrasound in mares treated with FSH in the ovarian stroma: evaluation of oocyte recovery rate}

\author{
Sergio Aguiar de Barros Vianna, ${ }^{*}$ José Frederico Straggiotti Silva, ${ }^{* *}$ Reginaldo da Silva Fontes ${ }^{* *}$
}

\begin{abstract}
Resumo
Um dos maiores problemas observados na eqüinotecnia se refere à otimização no número e precocidade de descendentes que uma égua pode produzir durante sua vida reprodutiva. A transferência de embriões em eqüinos associada às modernas técnicas de reprodução tais como: inseminação artificial com sêmen congelado, congelamento de embriões, punção de ovócitos in vivo e fertilização in vitro, possui grande potencial e aplicação prática para redução do intervalo entre gerações de eqüinos com pedigree de alto valor genético, além de permitir a multiplicação e conservação de espécies ameaçadas de extinção. Diante desta premissa, no intuito de contribuir com os estudos nesta área, utilizou-se neste experimento a técnica de punção folicular com uso de uma guia de biópsia e monitoramento ultra-sonográfico. Foi inoculado no estroma ovariano em um grupo de éguas $(n=10), 3 \mathrm{mg}$ armour de hormônio folículo estimulante (FSH- 1,5 ml de Folltropin), e no grupo controle $(\mathrm{n}=10) 1,5 \mathrm{ml}$ de solução salina. Após 24 horas os folículos ${ }^{3} 15 \mathrm{~mm}$ foram puncionados in vitro, obtendo-se um incremento na taxa de recuperação ovocitária significativo para os ovários tratados com FSH $(36,36 \%)$ quando comparados com os ovários tratados com solução salina $(10,52 \%)$. A técnica foi reproduzida in vivo e os resultados não foram os mesmos. Acredita-se que o aprimoramento da técnica com relação à realização de lavagens e o incremento de outras substâncias seja o ponto de viabilização para a obtenção de resultados similares in vivo e in vitro.
\end{abstract}

Palavras-chave: punção folicular, ultra-som, tratamento intra-folicular, éguas, FSH.

\begin{abstract}
One of the most important problems observed in horse production refers to optimize the number and prematurely of mare progeny that it can has during its reproductive life. The embryo transfer associated to modern techniques of equine reproduction, as well as: artificial insemination with frozen semen, frozen embryo, in vivo ovum pick-up, in vitro fertilization; have a great potential and practical applicability to reduce de space between generations in horses with high genetic value of pedigree, besides that allow a multiplication and conservation of species in extinguishing. To contribute for farther studies in this area, it was used in this experiment a follicular puncture technique with a use of biopsy guide ultrasound monitoring. It was inoculated in ovarian of a mare group ( $n=10) 3 \mathrm{mg}$ armour of follicular stimulating hormone (FSH) 1,5 ml Folltropin and in the control group $(\mathrm{n}=10) 1,5 \mathrm{ml}$ saline solution. After 24 hours the follicles ${ }^{3} 15 \mathrm{~mm}$ were punctured in vitro, resulting in a significant increase on oocyte recovery rate forth ovaries treated with FSH (36,36 \%) when compared with ovaries treated with saline solution $(10,52 \%)$. The technique was reproduced in vivo and the results were not the same. This believe that the improvement of the technique in relation to flushing and the development of other substances are the point to obtain similar results in vivo and in vitro.
\end{abstract}

Keywords: follicular puncture, ultrasound, mares, follicular treatment, FSH.

Introdução

A idéia đaa utilização do hormônio folículo estimulante intraovarianamente partiu de uma necessidade de reproduzir in vivo um ambiente de maturação ovocitária utilizado in vitro por Shabpareh et al. (1993), para que nos proporcionasse um incremento na taxa de recuperação dos mesmos, uma vez que sua maturação implicaria uma expansão de suas células do cumulus e um conseqüente incremento na taxa de recuperação destes.
A coleta de ovócitos in vivo pode ser realizada por uma aspiração de folículos grandes após sua exposição através de uma incisão no flanco (Vogelsang et al., 1986); passando a agulha pelo flanco após a estabilização manual do ovário pelo reto (Carnevale et al., 1988, Palmer et al., 1987), após uma ovariectomia (Hinrichs e Kenney, 1987, Hinrichs et al., 1990) ou utilizando-se uma agulha guiada por imagens ultra-sonograficas através do flanco (Palmer et al.,1986, King et al.,1987).

\footnotetext{
*Médico-veterinário, professor doutor do Laboratório de Zootecnia e Nutrição Animal (LZNA) do Centro de Ciência e Tecnologia (CCTA) da Universidade Estadual do Norte Fluminense (UENF) - Av. Alberto Lamego,2000 Bairro: Horto - Campos dos Goytacazes - RJ Cep:28015-620 - email: vianna@uenf.br

**Médico-veterinário, professor doutor do laboratório de Melhoramento Genético Animal(LMGA) do Centro de Ciência e Tecnologia (CCTA) da Universidade Estadual do Norte Fluminense (UENF)
} 
Com as coletas feitas via flanco em punções através de uma agulha, com os ovários manuseados e conduzidos pela mão via retal, obteve-se uma recuperação de $68 \%$ a $71 \%$ de ovócitos em folículos pré-ovulatórios (Palmer et al., 1987; McKinnon et al, 1987). No experimento de Palmer et al. (1987) as éguas submetidas à punção folicular foram tratadas com hCG (gonadotrofina coriônica humana) ou extrato de pituitária eqüina, e segundo McKinnon et al. (1987), os folículos foram monitorados diariamente via imagens ultra-sonográficas transretais, com objetivo de determinar o momento iminente da ovulação.

Palmer et al .(1987) e Mc Kinnon et al. (1987) mencionaram ainda em seus experimentos que a taxa de recuperação de ovócitos de folículos não estimulados ou imaturos foi de $0 \%$ a $16 \%$, o que, segundo Hinrichs et al. (1990), seria o reflexo da grande aderência do ovócito às células do cumulus na parede do folículo, dificultando sua recuperação.

Recentemente a aspiração de folículos por meio de guia de biópsia monitorada por imagens ultra-sonográficas transvaginais foi adaptada para éguas (Bruck et al., 1992; Meintjes et al., 1994).

A recuperação de ovócitos feitas geralmente em éguas vazias durante o diestro e/ou em éguas prenhes, as taxas de recuperação dos ovócitos provenientes desta técnicas de punção folicular são muito baixas $(<35 \%)$ e a percentagem de ovócitos obtidos com células do cumulus incompletas é muito grande (Cook et al., 1993; Dippert et al., 1994).

Mais recentemente Bogh et al. (2002), obtiveram em seu experimento resultados mais promissores, aspirando folículos de diferentes diâmetros, no estro e no diestro, e obtendo boas taxas de recuperação de oocitos, porém as taxas de maturação oocitárias foram muito ruins em oocitos provindos de folículos imaturos, uma vez que para a recuperação dos mesmos foram feitos cinco lavagens consecutivas dos folículos, o que causou grandes lesões nos complexos cumulus oocitos.

Hylan et al. (2002), vêm confirmar as informações fornecidas por Bogh et al. (2002), em que em seu trabalho só obtiveram boas taxas de recuperação em éguas com um efetivo resultado no tratamento com HCG (Gonadotrofina coriônica humana).

Em bovinos, a técnica de punção de ovócitos, seguida da maturação, fertilização e cultivo embrionário inicial in vitro, permite aumentar três vezes o número de bezerros vaca/ano, quando comparada com a transferência de embriões convencional (Peixer et al., 1996a; Peixer et al., 1996b ).

A explicação para estes diferentes resultados obtidos em bovinos e eqüinos se deve em grande parte na grande diferença histológica entre as estruturas celulares reprodutivas destas espécies. No ovócito bovino a distância entre a lâmina basal e as células do cumulus é bem maior, em média 136,83 $\mu \mathrm{m}$ e em éguas de 61,63 $\mu \mathrm{m}$. Em geral, a média de camadas de células do cumulus é menor em eqüinos do que em bovinos (Hawley et al., 1995).

$\mathrm{Na}$ descrição da técnica de punção folicular monitorada por imagens ultra-sonográficas transvaginais, Carnevale e Ginther (1993) administraram 10 minutos antes da aspiração uma injeção I.V. de $0,1 \mathrm{mg} / \mathrm{kg}$ de p.v. de brometo de propantheline para relaxar o reto, e cinco minutos antes realizaram uma sedação com 0,7mg/kg de p.v. de xilazina e 0,02mg/kg de p.v. de tartarato de butorfanol. Brück et al. (1997) utilizaram em cada égua uma injeção intravenosa de $10 \mu \mathrm{g} / \mathrm{kg}$ de p.v. de cloridrato de detomidina, $10 \mu \mathrm{g} / \mathrm{kg}$ de p.v. de sulfato de atropina, $30 \mu \mathrm{g} / \mathrm{kg}$ de p.v. de acepromazina I.V. e após cinco minutos $30 \mu \mathrm{g} / \mathrm{kg}$ de p.v. de tartarato de butorfanol. Além destes medicamentos foram utilizados também analgésicos e 0,15 mg/ $\mathrm{kg}$ de p.v. de brometo de propantheline para um relaxamento do reto.

Gastal e Ginther (1995) inocularam 1000 UI de eCG no fluido folicular, utilizando uma guia de biópsia acoplada a um ultrasom e verificaram que os folículos puncionados apresentavam indicações ultra-sonográficas de luteinização.

Objetivou-se no presente estudo coletar e avaliar morfologicamente ovócitos puncionados de éguas estimuladas com aplicação de FSH intra-ovariano, simplificando ao máximo a técnica de punção folicular e procurando manter ou melhorar os resultados relacionados com a quantidade e qualidade dos ovócitos coletados.

\section{Material e métodos}

Este experimento foi realizado em duas etapas: na primeira, inoculou-se no grupo tratado, por via intra-ovariana, FSH em no grupo controle de éguas e solução salina.

Nesta primeira etapa foram utilizadas para o experimento 20 éguas mestiças (Equus caballus) de diversas regiões do estado de Minas Gerais, Goiás e São Paulo, compreendidas num raio máximo de $600 \mathrm{Km}$ da Cidade de Araguari - MG, de propriedade da divisão de frigoríficos da Pomar S/A industrial e comercial (Araguari - MG). A idade estimada das éguas foi de dois a 20 anos, obtida por meio de indícios morfológicos e anatômicos, dentre estes a evolução dentária. Todas as éguas foram criadas extensivamente e estavam em estado sanitário e ginecológico sadios.

Inicialmente as 20 éguas foram escolhidas de um total de 300 animais, utilizando-se dois critérios básicos: 1. estado físico externo (selecionando as que apresentavam uma meIhor aparência geral, sem nenhum ferimento ou escoriação e peso ${ }^{3}$ a 250kg), 2. estado ginecológico (selecionando, por meio de exame ultra-sonográfico via retal, as que estavam com maior atividade ovariana e com pelo menos um de seus ovários de tamanho nunca inferior ao de uma noz).

As éguas foram conduzidas e contidas por intermédio de uma contenção mecânica simples, utilizando-se de um cabresto de corda e um boxe para exame ginecológico, complementando-se com uma contenção derivativa através de um cachimbo colocado na comissura labial superior. Os animais foram sedados com acepromazina I.V. na dose de $0,005 \mathrm{mg} / \mathrm{kg}$ de p.v. (Acepran ${ }^{\circledR} 1 \%$ da Univet S.A. Ind. Veterinária) cinco minutos antes da punção. Uma vez contidas, as éguas foram submetidas inicialmente a um esvaziamento do reto, em seguida foram higienizadas na região perineal com água e solução desinfetante. A anestesia do fundo de saco vaginal foi feita, introduzindo-se localmente, por meio de uma seringa conduzida pela mão enluvada, uma solução 1:1 de gel hidroalcoólico e cloridrato de lidocaína com norepinefrina (Xylocaina $^{\circledR}$ a $2 \%$ com norepinefrina da Astra Química e Farmacêutica Ltda.). 
No estroma ovariano foi inoculado, por meio de uma agulha de diâmetro de $18 \mathrm{G}$ e $6 \mathrm{~cm}$ de comprimento, acoplada a uma guia de biópsia, um volume de $1,5 \mathrm{ml}(30 \mathrm{mg})$ de $\mathrm{FSH}$ de origem suína (Folltropin ${ }^{\circledR}$ - V, Vetrepharm Inc.) em 10 éguas (grupo A) e 1,5 ml de solução salina em outras 10 éguas (Grupo B, controle). Após 24 horas as éguas foram abatidas, os ovários foram coletados e os folículos puncionados in vitro para recuperação e classificação dos ovócitos. Apenas um dos ovários de cada égua, tanto no grupo A com no grupo B, foi tratado. Os ovários trabalhados foram aqueles que apresentaram folículos com tamanho superior a $15 \mathrm{~mm}$ de diâmetro. A aspiração folicular foi feita por uma seringa de 20 ou $50 \mathrm{ml}$ acoplada a uma agulha de $40 \times 12 \mathrm{~mm}$ e em seguida 0 líquido folicular foi depositado em um tubo cônico de $50 \mathrm{ml}$ para posterior recuperação do ovócito. Foi realizada, após a aspiração dos folículos, uma lavagem do antro no intuito de se obter os ovócitos desejados, com uma maior margem de segurança,. Realizaram-se três lavagens em cada folículo e estes foram também massageados para uma melhor liberação do ovócito. A solução utilizada, segundo MEINTJES et al. (1995), consistiu de heparina, soro fetal bovino, penicilina-G e estreptomicina.

O equipamento ultra-sonográfico utilizado no procedimento de administração de FSH ou solução salina no estroma ovariano da primeira etapa e para a aspiração folicular in vivo nas éguas da segunda etapa foi um Scanner 200 Vet $^{\circledR}$ fabricado pela Pie Medical Equipment B.V. com um transdutor setorial multiângulo bifreqüencial de 5 \& 7,5 Mhz e uma guia de biópsia acoplada a uma agulha de lúmen simples, calibre de 18 gauge e comprimento de $6 \mathrm{~cm}$, com bisel protegido.

$\mathrm{Na}$ segunda etapa, de posse dos resultados da primeira etapa, foi reproduzido o tratamento com FSH no estroma ovariano em 12 éguas e a punção folicular, guiada por ultra-som, feita in vivo. A agulha acoplada através de um conector de aço inox a uma seringa de lavagem e uma bomba de sucção exerceu um vácuo variável em torno de $200 \mathrm{~mm}$ de $\mathrm{Hg}$ para a aspiração do folículo. O tubo de aço inox com agulha em sua posição craneal foi inserido no tubo guia, sendo então incorporado junto ao transdutor do equipamento ultra-sonográfico.

Uma vez o ovário fixado, manualmente, via transretal, a aguIha de biópsia foi guiada até o folículo. Para este procedimento, o transdutor e a agulha para punção do folículo foram recobertos por um filme e em seguida aplicado um lubrificante estéril. $\mathrm{O}$ transdutor foi inserido na vagina da égua e no interior conduzido até próximo ao ovário a ser inoculado. $\mathrm{O}$ ovário foi conduzido e fixado no fundo de saco vaginal por tração manual via retal, onde estava posicionado para a inoculação no ovário.

O líquido aspirado foi colocado em tubos de ensaio estéreis de plástico e filtrados com filtro de coleta de embriões, os ovócitos foram observados em um estereomicroscópio com aumento de $40 \mathrm{X}$. A qualidade dos ovócitos foi julgada de acordo com o número de camadas e degeneração das células da granulosa, transparência e homogeneidade do citoplasma e presença de vacúolos, segundo Parveliet et al. (1993).

Para a análise estatística utilizamos o teste qui-quadrado em uma tabela de contingência $2 \times 2$.

\section{Resultados e discussão}

A comparação dos resultados obtidos nos tratamentos com FSH e solução salina estão apresentados nas Tabelas 1, 2 e 3.

Tabela 1: Efeito da estimulação in vivo com FSH intra-ovariano na punção folicular pós-morte de ovários de éguas demostrados através do teste qui-quadrado em uma tabela de contingência $2 \times 2(P<0,05)$, grupo controle inoculação de solução salina.

\begin{tabular}{c|c|c|c} 
& $\mathbf{T}_{\mathbf{1} \text { (FSH) }}$ & $\mathbf{T}_{\text {2(Sol.salina) }}$ & \\
\hline $\mathbf{R}$ & $13(10)$ & $2(5)$ & 15 \\
\hline$\tilde{\mathbf{n}} \mathbf{R}$ & $39(42)$ & $24(21)$ & 63 \\
\hline & 52 & 26 & 78
\end{tabular}

$\mathbf{T}_{1}=$ Tratamento $1 ; \mathbf{T}_{2}=$ Tratamento $2 ; \mathbf{R}=$ Ovócitos recuperados; $\tilde{n} \boldsymbol{R}=$ Óvócitos não recuperados

Tabela 2: Efeito local da estimulação in vivo com FSH intraovariano na punção folicular pós-morte de ovários de éguas demostrados através do teste qui-quadrado em uma tabela de contingência $2 \times 2(P<0,05)$, grupo controle inoculação de solução salina.

\begin{tabular}{c|c|c|c} 
& $\mathbf{T}_{\mathbf{1}(\mathrm{FSH})}$ & $\mathbf{T}_{\mathbf{2}}$ (Sol.salina) & \\
\hline $\mathbf{R}$ & $12(20,94)$ & $2(5,11)$ & 14 \\
\hline$\tilde{\mathbf{n}} \mathbf{R}$ & $21(24,11)$ & $17(13,88)$ & 38 \\
\hline & 33 & 19 & 52
\end{tabular}

$\mathbf{T}_{1}=$ Tratamento $1 ; \mathbf{T}_{2}=$ Tratamento $2 ; \mathbf{R}=$ Ovócitos recuperados; $\tilde{\mathbf{n}} \mathbf{R}=$ Ovócitos não recuperados

A taxa de recuperação não apresentou diferença significativa quando analisado o total de ovócitos recuperados sem levar em conta o ovário em que foi aplicado o tratamento (Tabela 1), o que significa que o resultado na recuperação dos ovócitos, levando em consideração os dois ovários, não depende do tratamento. Nos dados da Tabela 2, analisando o local da aplicação, ou seja, apenas os dados provenientes dos ovários tratados e não seu homólogo, os resultados apresentaram uma diferença significativa $(\mathrm{P}<0,05)$ quanto ao uso do FSH ou da solução salina na recuperação de ovócitos, portanto, significa que o resultado in loco depende do tratamento utilizado. Foi possível observar que os ovários tratados com FSH aumentaram de tamanho e quantitativamente observouse que o número de folículos puncionados foi maior com o uso do FSH, apesar do número de éguas utilizadas ter sido o mesmo para os dois tratamentos. Observou-se ainda que, com o uso do tratamento intra-ovariano de $\mathrm{FSH}$, a qualidade dos ovócitos foi melhor. 
Tabela 3: Efeito da estimulação com FSH intra-ovariano (grupo controle: inoculação de solução salina) na classificação dos ovócitos.

\begin{tabular}{ccc}
\hline $\begin{array}{c}\text { CLASSIFICAÇÃO } \\
\text { DOS OVÓCITOS } \\
\text { RECUPERADOS }\end{array}$ & COM FSH & COM SOL. SALINA \\
\hline Classe I & 6 & 0 \\
\hline Classe II & 4 & 0 \\
\hline Classe III & 2 & 1 \\
\hline Classe IV & 1 & 1 \\
\hline Total & 13 & 2 \\
\hline
\end{tabular}

Outro dado qualitativo bastante importante foi relativo ao experimento realizado in vivo, na segunda etapa deste estudo, em que se inoculou o FSH no estroma ovariano, 24 horas antes dos folículos serem puncionados, e em nenhuma das 12 éguas puncionadas obteve-se a recuperação dos ovócitos.

De forma diferente e simplificada em relação à utilizada por Carnevale e Ginther (1993) e Brück et al. (1997) para contenção e preparo para execução da técnica de punção folicular e tratamento intra-ovariano, utilizou-se, no presente experimento, uma contenção mecânica simples, uma contenção derivativa, e uma contenção farmacológica; em seguida, foi realizada a anestesia do fundo de saco vaginal. Todos estes procedimentos foram eficientes na manipulação e punção folicular deste experimento. Procedeu-se, em nossos ensaios experimentais, também a técnica segundo Brück et al. (1997) e não foi verificada diferença na execução em comparação com o método utilizado neste experimento. Acredita-se que a simplificação na realização da técnica é uma necessidade. Foi implementado o uso do cachimbo como descrito anteriormente, o que pode ter sido decisivo na contenção, substituindo algumas drogas sedativas como a detomidina, permitindo, assim, a redução de custos e agilidade de execução.

\section{Referências}

BOGH,I.G.; BÉZARD, J.; DUCHAMP, G.; BALTSEN, M.; GERARD, N.; DAELS, P.; GREVE,T. Pure preovulatory follicular fluid promotes in vitro maturation of in vivo aspirated equine oocytes. Theriogenology, v. 57, p. 1765-1779, 2002.

BRÜCK, I.; RAUN, K.; SYNNESTVEDT, B.; GREVE, T. Follicle aspiration in the mare using a transvaginal ultrasound-guided technique. Equine Vet. J., v. 24, p. $58-59,1992$.

BRÜCK, I.; SYNNESTVEDT, B.; GREVE, T. Repeated transvaginal oocyte aspiration in unstimulated and $\mathrm{FSH}$-treated mares. Theriogenology. v. 47, p. 1157-1167, 1997.

CARNEVALE, E. M.; GINTHER, O. J. Use of a linear ultrasonic transducer for the transvaginal aspiration and transfer of oocytes in the mare. Journal of Equine Veterinary Science. v. 13, p. 331-333, 1993.

CARNEVALE, E. M.; McKINNON, A. O.; SQUIRES, E. L. Effect of preovulatory follicular fluid aspiration upon luteal fuction in the mare. Theriogenology, v. 29, p. 231, 1988.

COOK, N. L.; SQUIRES, E. L.; RAY, B. S.; JASKO, D. J. Transvaginal ultrasound-guided follicular aspiration of equine oocytes. Equine Vet. Journal, v. 15, p. 71- 74, 1993.

DIPPERT, K. D.; RAY, B. S.; SQUIRES, E. L. Maximising ultrasound-guided retrieval of equine oocytes. Theriogenology, v. 41, p. 190, 1994.

GASTAL, E.L.; GINTHER, O.J. Ultrasound-guided intrafollicular treatment in mares. Theriogenology, v. 44, p. 1027-1037, 1995.
Da mesma forma, neste experimento, como verificado por Palmer et al. (1987) e McKinnon et al. (1987), a aspiração de folículos imaturos teve uma taxa de recuperação dos ovócitos muito baixa, na ordem de 0 a 16\%. A recuperação de ovócitos feita em éguas vazias durante o diestro e/ou em éguas prenhes mostrou taxas de recuperação dos ovócitos provenientes de técnicas de punção folicular muito baixas (<35\%), além da percentagem de ovócitos obtidos com células do cumulus incompletas ser muito grande (Brück et al. 1992 e Meintjes et al., 1994). No presente estudo o resultado encontrado na taxa de recuperação in vivo foi de $0 \%$. Diante destes fatos, é importante ressaltar que as taxas de recuperação ovocitária estão em consonância com a literatura citada anteriormente, e o uso do FSH intra-ovariano foi benéfico na otimização desta, como mostram os resultados in vitro. Com relação à taxa de recuperação obtida in vivo não ter repetido as taxas obtidas in vitro, prende-se muito provavelmente ao fato de que in vivo não foi possível realizar o número de lavagens realizados in vitro, que foi da ordem de cinco a seis, causados pela dificuldade de conter o ovário imóvel diante da agulha de lavagem. Outro fato que acredita-se ter contribuído da mesma forma para este resultado in vivo foi, também, a impossibilidade de, durante a punção, realizar-se uma massagem no folículo puncionado para facilitar a liberação do ovócito.

A execução da técnica de inoculação de FSH intra-ovariano permitiu um incremento na taxa de recuperação de ovócitos, porém, mais estudos são necessários para que a obtenção dos resultados sejam os mesmos in vitro e in vivo. A etapa das lavagens da cavidade antral deve ser melhor trabalhada, possivelmente acrescentando substancias de lavagem que facilitem a recuperação dos ovócitos. Portanto, a técnica de punção folicular guiada por ultra-sonografia, em éguas com o uso do tratamento intra-ovariano de FSH, poderia ser uma alternativa viável e capaz de solucionar alguns problemas oriundos da dificuldade de se aumentar a quantidade de descendentes de uma égua durante sua vida reprodutiva.

HAWLEY, L. R.; ENDERS, A. C.; HINRICHS, K. Comparison of equine and bovine oocyte-cumulus morfology within the ovarian follicle. Biol. Reprod. Mono, v.1, p. $243-252,1995$.

HYLAN, D.; KLUMPP, A. M.; SANSINENE, M.; LaCASE, R. J.; PACCAMONTI,D; DENNISTON, R. A.; GODKE, R. A. Oocyte production from naïve- and chronically-aspirated mares. Theriogenology, v. 58, p. 739-741, 2002.

HINRICHS, K.; KENNEY, D. F.; KENNEY, R. M. Aspiration of oocytes from mature and immature preovulatory follicles in the mare. Theriogenology, v. 34, p. 107-112, 1990.

HINRICHS, K.; KENNEY, R. M. A colpotomy procedure to increase oocyte recovery rates on aspiration of equine preovulatory follicles. Theriogenology, v. 27, p. 237, 1987.

KING, W. A.;BEZARD, J.;BOUSQUET, D.; PALMER, E.;BETTERIDGE, K. $J$. The meiotic stage of preovulatory oocytes in the mare. Genome, v. 29, p. 679-682, 1987.

MEINTJES, M.; BELLOW, M. S.;BROUSSARD, J. R.; PACCAMONTI, D.; EILTS, B. E.; GODKE, R. A. Repeated transvaginal ultrassound-guided oocyte retrieval from pregnant mares. Theriogenology, v. 41, p. 255, 1994.

MEINTJES, M.; BELLOW, M. S.; PAUL, J. B.;BROUSSARD, J. R.; LI, L. Y.; PACCAMONTI, D.; EILTS, B. E.; GODKE, R. A. Transvaginal ultrassound-guided oocyte retrieval from cyclic and pregnant horse and pony mares for in vitro fertilization. Biol. Reprod. Mono, v. 1, p. 281-292, 1995. 
PALMER, E.; DUCHAMP, G.;BEZARD, J.; MAGISTRINI, M.; KING, W. A.; BOUSQUET, D.; BETTERIDGE, K. J. Non-surgical recovery of follicular fluid and oocytes of mares. J. Reprod. Fertil, v. 35, p. 689-690, 1987.

PALMER, E.; DUCHAMP, G.; BEZARD, J.; MAGISTRINI, M.; KING, W. A.; BOUSQUET, D.; BETTERIDGE, K. J. Recovery of follicular fluid and oocytes of mares by non-surgical puncture of the preovulatory follicle. Theriogenology, v. 25, p. 178, 1986.

PARVELIET, J. M.; DE LOOS, F.; FAZELI, A. R.; BEVERS, M.; VOS, P. L. A. M.; PIETERSE, M.; TAVERNE, M.; COLENBRANDER, B. Transvaginal ultrassound-guided ovum pick up in the horse: recovery rate and oocyte quality. J. Reprod. Fert. Abstract Series, v. 12, p. 40, 1993.

PEIXER, M. A. S.; FONTES, R. R.; LUNA, N. M.; CAMARA, J. U.; RUMPF, $R$. Produção in vitro de embriões a partir de ovócitos coletados de animais vivos em condições de campo. Arq. Fac. Vet. UFRGS, v. 24, p. 230, 1996a.

PEIXER, M. A.S.; RUMPF, R.;DEBEM, A. R.; QUEIROZ, L. M. V. Produção de embriões e gestações a partir de ovócitos recuperados por ultrasonografia em fêmeas bovinas superovuladas. Arq. Fac. Vet. UFRGS, v. 24, p. 231, 1996b.

SHABPAREH, V.; SQUIRES, E. L.; SEIDEL, G. E.; JARO, J. R. Methodos for collecting and maturing equine oocytes in vitro. Theriogenology, v. 40, p. 1161-1175, 1993.

VOGELSANG, M. M.; KRAEMER, D. C.; BOWEN, M. J.;POTTER, G. D. Recovery of equine follicular oocytes by surgical and non-surgical techniques. Theriogenology, v. 25, p. 208, 1986. 